\title{
Konkomitante Einnahme von Medikamenten erhöht das Risiko für Frakturen
}

Abtahi S et al. Concomitant use of oral

glucocorticoids and proton pump inhibitors and

risk of osteoporotic fractures among patients

with rheumatoid arthritis: a population-based

cohort study. Ann Rheum Dis 2021; 80: 423-431

Patienten mit Rheumatoider Arthritis (RA), die orale Glucocorticoide einnehmen, haben häufig eine reduzierte Knochenmineraldichte und weisen ein erhöhtes Risiko für Frakturen auf. Oftmals werden mehrere Medikamente für verschiedene Indikationen auf einmal eingenommen. Abtahi et al. untersuchten den Zusammenhang zwischen der gleichzeitigen Einnahme von Glucocorticoiden und Protonenpumpeninhibitoren und dem Risiko für Frakturen bei RAPatienten.

Die Forscher stellten im Rahmen der retrospektiven Kohortenstudie fest, dass es eine Interaktion bezüglich des Risikos für osteoporotische Frakturen bei gleichzeitiger Anwendung von oralen Glucocorticoiden und Protonenpumpenhemmern gibt. Die Arbeitsgruppe um Abtahi werteten Daten aus der Datenbank des Clinical Practice Research Datalink (CPRD), eine der größten Datenbanken der Welt für Primärversorgungsdaten, aus. Die Studienkohorte umfasste Erwachsene im Alter von mindestens 50 Jahren, bei denen zwischen Januar 1997 und Dezember 2017 eine RA diagnostiziert wurde. Das Datum der ersten RA-Diagnose während der gültigen Datenerfassung definierten die Wissenschaftler als Indexdatum. Ab diesem Datum erhoben die Forscher, ob und zu welchen Zeitpunkten die Patienten orale Glucocorticoide und Protonenpumpenhemmer einnahmen und welche Dosierung die Patienten verschrieben bekommen hatten. Als Endpunkt dieser Studie untersuchten die Experten die erste osteoporotische Fraktur an Hüfte, Wirbel, Humerus, Unterarm, Becken oder Rippen nach dem RA-Indexdatum. Als potenzielle Störvariablen nahmen die Forscher den Body-Mass-Index (BMI), Raucherstatus und Alkoholkonsum mit in die statistische Analyse auf. Neben dem Alter und der Kranken- 
geschichte ermittelten die Wissenschaftler das Auftreten zahlreicher Komorbiditäten.

Die Studienpopulation umfasste insgesamt 12.351 RA-Patienten. Die parallele Anwendung oraler Glucocorticoide und Protonenpumpeninhibitoren bei Patienten mit RA war mit einem 1,6-fach erhöhten Risiko für osteoporotische Frakturen verbunden. Sowohl die alleinige Einnahme oraler Glucocorticoide als auch die Einnahme von Protonenpumpenhemmern war mit einem 1,2-fach erhöhten Risiko für Frakturen verbunden. Patienten, die die Einnahme beider Medikamente für mehr als 6 Monate unterbrochen hatten, wiesen keine signifikante Erhöhung des osteoporotischen Frakturen-Risikos mehr auf. Eine parallele Einnahme von Kalzium und Vitamin D reduzierte das Risiko für eine Fraktur. Die Forscher beobachteten bei gleichzeitiger Anwendung von oralen Glucocorticoiden und Protonenpumpeninhibitoren ein 1,5fach erhöhtes Risiko für Hüftfrakturen, ein 2,8-fach erhöhtes Risiko für klinische Wirbelfrakturen, ein 2,5-fach erhöhtes Risiko für Beckenfrakturen und ein 4-fach erhöhtes Risiko für Rippenfrakturen. Das Risiko für eine Fraktur des Oberarmes oder des Unterarmes erwies sich als nicht erhöht.

\section{FAZIT}

Die parallele Anwendung von oralen Glucocorticoiden und Protonenpumpeninhibitoren erhöht das Risiko osteoporotischer Frakturen bei RA-Patienten. In Anbetracht der steigenden Lebenserwartung und des hohen Konsums von Protonenpumpeninhibitoren bei älteren RA-Patienten sollte eine Frakturrisikoabschätzung in Betracht gezogen werden, wenn einem Patienten mit RA gleichzeitig orale Glucocorticoiden und Protonenpumpeninhibitoren verschrieben werden, so die Experten.

Dr. Maddalena Angela Di Lellis, Tübingen 